PROCEEDINGS OF THE AMERICAN MATHEMATICAL SOCIETY

Volume 124, Number 9, September 1996

\title{
A WOLFF-DENJOY THEOREM FOR INFINITELY CONNECTED RIEMANN SURFACES
}

\author{
FINNUR LÁRUSSON
}

(Communicated by Theodore W. Gamelin)

\begin{abstract}
We generalize the classical Wolff-Denjoy theorem to certain infinitely connected Riemann surfaces. Let $X$ be a non-parabolic Riemann surface with Martin boundary $\Delta$. Suppose each Martin function $k_{y}, y \in \Delta$, extends continuously to $\Delta \backslash\{y\}$ and vanishes there. We show that if $f$ is an endomorphism of $X$ and the iterates of $f$ converge to the point at infinity, then the iterates converge locally uniformly to a point in $\Delta$. As an application, we extend the Wolff-Denjoy theorem to non-elementary Gromov hyperbolic covering spaces of compact Riemann surfaces. Such covering surfaces are of independent interest. Finally, we use the theory of non-tangential boundary limits to give a version of the Wolff-Denjoy theorem that imposes certain mild restrictions on $f$ but none on $X$ itself.
\end{abstract}

Introduction. The classical theorem on the iteration of endomorphisms of the unit disc $\mathbb{D}$, i.e., holomorphic maps from $\mathbb{D}$ into itself, is the theorem of Wolff and Denjoy of 1926. It states that if such a map $f$ is not an elliptic automorphism of $\mathbb{D}$, then the iterates $f^{n}$ converge locally uniformly to a point in the closed unit disc $\overline{\mathbb{D}}$. The following theorem of Heins [Hei] seems to be the strongest available generalization of the Wolff-Denjoy theorem to arbitrary Riemann surfaces.

Theorem (Heins). Let $f$ be an endomorphism of a Riemann surface $X$ covered by $\mathbb{D}$. Then one of the following holds:

(1) The iterates of $f$ converge locally uniformly to a point in $X$. This point is the unique fixed point of $f$.

(2) $f$ is an automorphism of $X$ of finite order.

(3) $X$ is $\mathbb{D}, \mathbb{D} \backslash\{0\}$ or an annulus, and $f$ is an irrational rotation, so a subsequence $\left(f^{n_{k}}\right)$ converges locally uniformly to the identity.

(4) The iterates of $f$ converge locally uniformly to an end of $X$.

Moreover, in case (4), if $X$ is a smoothly bounded domain in a compact Riemann surface, then the iterates of $f$ converge locally uniformly to a boundary point of $X$.

Received by the editors March 3, 1995.

1991 Mathematics Subject Classification. Primary 30F25, 32H50.

Key words and phrases. Riemann surface, endomorphism, iteration, Wolff-Denjoy theorem, Martin boundary, Picard existence principle, non-tangential limit, covering space, hyperbolic group, Gromov space.

This work was supported in part by the Icelandic Council of Science and by the U.S. National Science Foundation under grant no. DMS-9400872.

(c)1996 American Mathematical Society 
Suppose now that $X$ is a non-parabolic Riemann surface (meaning that $X$ has a Green function), so the Martin boundary of $X$ is defined. For the theory of the Martin boundary, see [CC2] or [Has]. The Martin compactification is finer than the end compactification. In fact, the connected components of the Martin boundary correspond to the ends of $X$. It is natural to ask whether the iterates of an endomorphism of $X$ always converge to a point of the Martin boundary in case (4) above. As far as I know, this is an open question for infinitely connected surfaces. In this paper, we give an affirmative answer for surfaces $X$ that satisfy what we call the Picard existence principle, a simple and explicit condition on the Martin functions of $X$. It holds in particular if $X$ is regular and satisfies a boundary Harnack principle.

This enables us to extend the Wolff-Denjoy theorem to Galois covering spaces of compact Riemann surfaces whose covering group is non-elementary hyperbolic in the sense of Gromov. It is a non-trivial fact, due to Ancona [Anc], that such covering surfaces satisfy the Picard existence principle. These surfaces are infinitely connected, except for the disc itself. They have come up in investigations of covering spaces of projective algebraic manifolds related to the Shafarevich conjecture and turned out to have interesting function-theoretic properties; see [Lár]. Examples are given by connected Riemann surfaces in the open unit ball in $\mathbb{C}^{n}, n \geq 2$, that are preimages of smooth curves in compact quotients of the ball.

Notation. Let $X$ be a non-parabolic Riemann surface with Poincaré distance $d$. Let $G_{x}=G(x, \cdot)$ be the Green function with pole $x \in X$. Let $\Delta$ be the Martin boundary of $X$ with respect to a fixed base point $a \in X, \bar{X}=X \cup \Delta$ be the Martin compactification, and $k_{y}$ be the Martin function with pole $y \in \Delta$. It is the limit of $G(\cdot, z) / G(a, z)$ as $z \rightarrow y$. We define the following property that $X$ may or may not have.

Picard existence principle (PEP). Each Martin function $k_{y}$ extends continuously to $\Delta \backslash\{y\}$ and vanishes there.

Let us give a sufficient condition for PEP to hold. Suppose $X$ is regular, meaning that the Dirichlet problem can be solved for all continuous functions on $\Delta$. Let $y \in \Delta$ have a decreasing neighbourhood basis $V_{n}, n \in \mathbb{N}$. Let $u_{n}$ be a non-negative continuous function on $\Delta$, supported on $V_{n}$, whose harmonic extension $h_{n}$ has $h_{n}(a)=1$. Then

$$
h_{n}(x)=\int_{\Delta} k_{z}(x) u_{n}(z) \sigma(z), \quad x \in X,
$$

where $\sigma$ is the harmonic measure on $\Delta$. By passing to a subsequence, we may assume that the probability measures $u_{n} \sigma$ converge in the weak-star topology to a measure $\mu$ on $\Delta$. Clearly, $\mu$ is supported on $y$, so $\mu=\delta_{y}$ and $h_{n} \rightarrow k_{y}$ pointwise (and hence locally uniformly) by the integral formula.

This shows that PEP holds for a regular surface $X$ if the space of positive harmonic functions on $X$ that vanish on a given open set in $\Delta$ is closed in the locally uniform topology. Hueber [Hue] has shown that this is equivalent to the following boundary Harnack principle.

Boundary Harnack principle. For any open subsets $U$ and $V$ of $\bar{X}$ with $\bar{V} \subset U$ and any $x \in X$, there is $c>0$ such that

$$
h \leq c h(x) \quad \text { on } V \cap X
$$


for all positive harmonic functions $h$ on $X$ that vanish continuously on $U \cap \Delta$.

We now state our main result, which strengthens Heins' theorem. The method of proof is borrowed from [Hei].

Theorem 1. Let $X$ be a non-parabolic Riemann surface satisfying PEP. If the iterates of an endomorphism $f$ of $X$ converge to the point at infinity, then the iterates converge locally uniformly to a point in the Martin boundary of $X$.

Note that by Heins' theorem, it suffices to assume merely that the orbit $\left\{f^{n}(x)\right\}$ of some point $x \in X$ is unbounded. Note also that if $f^{n} \rightarrow \infty$ pointwise, then $f^{n} \rightarrow \infty$ locally uniformly since $f$ shrinks the Poincaré distance.

Before proceeding to the proof, we need to establish the following geometric property of the Martin compactification. It says that distinct Martin boundary points are infinitely far apart in the Poincaré metric.

Lemma. Let $X$ be a non-parabolic Riemann surface satisfying PEP, and let $p$ and $q$ be distinct points in $\Delta$. For every $M>0$ there are neighbourhoods $U$ of $p$ and $V$ of $q$ in $\bar{X}$ such that

$$
d(U \cap X, V \cap X)>M .
$$

Proof. Otherwise, there are sequences $x_{n} \rightarrow p$ and $y_{n} \rightarrow q$ in $X$ such that $d\left(x_{n}, y_{n}\right)$ is bounded. Then

$$
k_{q} \leq c k_{p}
$$

for some $c>0$ by [CC1]. Hence, by PEP, $k_{q}$ vanishes continuously on all of $\Delta$, so $k_{q}=0$, which is absurd.

Proof of Theorem 1. We may assume that $\Delta$ contains at least two points; otherwise there is nothing to prove. Then PEP implies that $G_{a}(x) \rightarrow 0$ as $x \rightarrow \infty$.

Choose $p \in X$ such that $p(n)=f^{n}(p) \neq a, n \geq 0$. Since $G_{a}(p(n)) \rightarrow 0$, there is a subsequence $(n(k))$ such that

$$
G_{a}(p(n(k)))>G_{a}(p(n(k)+1)) .
$$

Furthermore, there is a subsequence $(n(k(j)))$ such that $p(n(k(j)))$ converges to a point $q \in \Delta$.

Now $G_{x}$ is characterized as the smallest positive superharmonic function $u$ on $X$ with $u(z)+\log |z|$ harmonic near $x$, where $z$ is a local coordinate centred at $x$. This implies that

$$
G(f(x), f(y)) \geq G(x, y), \quad x, y \in X
$$

Hence,

$$
G(f(x), p(n(k(j))+1)) \geq G(x, p(n(k(j)))),
$$

and

$$
\frac{G(f(x), p(n(k(j))+1))}{G(a, p(n(k(j))+1))} \geq \frac{G(x, p(n(k(j))))}{G(a, p(n(k(j))))} .
$$

The Poincaré distance on $X$ is shrunk by $f$, so the distance between $p(n(k(j)))$ and $p(n(k(j))+1)$ is bounded independently of $j$. Hence, $p(n(k(j))+1) \rightarrow q$ by the lemma.

Letting $j \rightarrow \infty$ in $\left(^{*}\right)$ now yields

$$
k_{q} \circ f \geq k_{q} .
$$


For any $x \in X$, we have $f^{n}(x) \rightarrow \infty$ and $k_{q}\left(f^{n}(x)\right) \geq k_{q}(x)>0$, so $f^{n}(x) \rightarrow q$ by PEP. By the lemma, this implies that $f^{n} \rightarrow q$ locally uniformly on $X$.

It is easy to see that Theorem 1 holds under various slightly different hypotheses. For instance, the above proof works with minor modifications if we assume that $G_{a}$ vanishes at infinity and for every $q \in \Delta$,

(1) $k_{q}$ is unbounded, and

(2) for every neighbourhood $U$ of $q$ in $\bar{X}, k_{q}$ is bounded on $X \backslash U$.

Ancona's work. Ancona [Anc] has obtained strong results about the interplay of geometry and potential theory on Riemannian manifolds that are Gromov hyperbolic, coercive and have bounded geometry. Among other things, he has shown that they satisfy PEP for the Laplace-Beltrami operator. His conditions are rather complicated to state precisely, so we content ourselves with the following statement.

Theorem (Ancona). Let $X$ be a Galois covering space of a compact Riemannian manifold with a non-elementary hyperbolic covering group. Then $X$ satisfies PEP.

Hyperbolic groups were introduced by Gromov in [Gro]. See also [CDP] and $[\mathrm{GH}]$. Gromov has suggested that such groups are in a sense generic. A group is hyperbolic if it is Gromov as a metric space in the word metric. This means that geodesic triangles in the Cayley graph are uniformly thin. A hyperbolic group is either almost cyclic or non-amenable, in which case it is called non-elementary. Fundamental groups of compact Riemannian manifolds of negative sectional curvature are examples of hyperbolic groups. A Riemannian manifold $X$ as in the theorem is a Gromov space. Also, $X$ is known to be regular and to have an infinite-dimensional space of bounded harmonic functions.

From Theorem 1 and the theorems of Heins and Ancona, we now obtain the following extension of the Wolff-Denjoy theorem.

Theorem 2. Let $X$ be a Galois covering space of a compact Riemann surface with a non-elementary hyperbolic covering group. Let $f$ be an endomorphism of $X$. Then one of the following holds:

(1) The iterates of $f$ converge locally uniformly to a point in the Martin compactification of $X$.

(2) $f$ is an elliptic automorphism of $X$. Then $f$ has finite order, unless $X=\mathbb{D}$ and $f$ is an irrational rotation.

If $f$ is an automorphism, then $f$ is called elliptic if the orbit $\left\{f^{n}(x)\right\}$ of some and hence every point $x \in X$ is bounded. This is the general definition for an isometry of a Gromov space.

Examples. Let $M$ be a projective algebraic manifold of dimension $n \geq 2$ with a hyperbolic fundamental group. The class of such manifolds contains all compact Kähler manifolds of negative sectional curvature, such as ball quotients. Also, if $M_{o}$ is in this class, then so is $M$ if one of the following holds:

(1) $M$ is an ample divisor in $M_{o}$.

(2) $M=M_{o} \times M^{\prime}$ with $M^{\prime}$ projective and $\pi_{1}\left(M^{\prime}\right)$ finite.

(3) There is a finite unbranched covering $M \rightarrow M_{o}$ or $M_{o} \rightarrow M$.

Suppose $M$ is embedded into some projective space, and let $C$ be the transverse intersection of $M$ by a linear subspace of codimension $n-1$. Then $C$ is a smooth curve in $M$. By the Lefschetz hyperplane theorem, $C$ is connected and the map 
$\pi_{1}(C) \rightarrow \pi_{1}(M)$ is surjective, which implies that the preimage $X$ of $C$ in the universal covering space of $M$ is connected. The Riemann surface $X$ is non-parabolic iff $\pi_{1}(M)$ is infinite, and then the Wolff-Denjoy theorem holds for $X$.

Final remarks. In view of Theorem 1, one might conjecture that if the iterates of an endomorphism of a non-parabolic Riemann surface $X$ converge to the point at infinity, then they converge locally uniformly to a point in the Martin boundary of $X$. Let us conclude by providing some further evidence for this conjecture.

We can lift an endomorphism $f$ of $X$ to an endomorphism $F$ of the universal covering $\mathbb{D}$. Suppose $f^{n} \rightarrow \infty$. Then $F^{n} \rightarrow \infty$, and by the original Wolff-Denjoy theorem, $F^{n} \rightarrow q \in \mathbb{T}=\partial \mathbb{D}$ locally uniformly in $\mathbb{D}$.

Observation. Let $X$ be a non-parabolic Riemann surface with Martin boundary $\Delta$ and universal covering map $\phi: \mathbb{D} \rightarrow X$. Let $f$ be an endomorphism of $X$ with a lifting $F: \mathbb{D} \rightarrow \mathbb{D}$ such that

(1) $F^{n} \rightarrow q \in \mathbb{T}$ non-tangentially, and

(2) $\phi$ has a non-tangential limit $p \in \Delta$ at $q$.

Then $f^{n} \rightarrow p$.

This is of course obvious, but the point is that no conditions are imposed on $X$, and conditions (1) and (2) on $f$ are, in a sense, generically satisfied, as we will now explain.

First, by a result of Cowen $[\mathrm{Cow}]$, if $F: \mathbb{D} \rightarrow \mathbb{D}$ is an endomorphism and $F^{n} \rightarrow q \in \mathbb{T}$, then $F^{n} \rightarrow q$ non-tangentially, unless $F$ is conjugate to a parabolic Möbius transformation on a fundamental set. A fundamental set is an $F$-invariant domain that eventually swallows any compact set under the action of $F$. Also, if the angular derivative

$$
F^{\prime}(q)=\lim _{r \rightarrow 1-} F^{\prime}(r q) \in(0,1]
$$

is not equal to 1 , then $F^{n} \rightarrow q$ non-tangentially. It is reasonable to interpret this as saying that $F^{n} \rightarrow q$ non-tangentially for the generic $F$.

As for (2), combining results from [CC2, Chapter 19] and [Has, p. 60], we obtain the following statement.

Theorem. The universal covering map $\phi: \mathbb{D} \rightarrow X$ has a non-tangential limit in $\Delta$ at almost every point of $\mathbb{T}$.

Let us note that in general there are points in $\mathbb{T}$ where $\phi$ does not have a nontangential limit in $\Delta$. Conical limit points of the covering group $G$, for instance fixed points of hyperbolic elements of $G$, are examples of such points. Hence, the theorem directly implies the well-known fact that the conical limit set of $G$ has measure zero.

\section{REFERENCES}

[Anc] A. Ancona, Théorie du potentiel sur les graphes et les variétés, École d'éte de probabilités de Saint-Flour XVIII - 1988, Lecture notes in mathematics, vol. 1427, SpringerVerlag, Berlin, 1990, pp. 1-112. MR 92g:31012

[CC1] C. Constantinescu and A. Cornea, Über den Martinschen idealen Rand einer Riemannschen Fläche, Rev. Math. Pures Appl. 5 (1960), 21-25. MR 24:A1388

[CC2] Ideale Ränder Riemannscher Flächen, Ergebnisse der Mathematik und ihrer Grenzgebiete, N. F., Band 32, Springer-Verlag, Berlin, Göttingen, Heidelberg, 1963. MR 28:3151 
[CDP] M. Coornaert, T. Delzant and A. Papadopoulos, Géométrie et théorie des groupes, Lecture Notes in Mathematics, vol. 1441, Springer-Verlag, Berlin, 1990. MR 92f:57003

[Cow] C. C. Cowen, Iteration and the solution of functional equations for functions analytic in the unit disc, Trans. Amer. Math. Soc. 265 (1) (1981), 69-95. MR 82i:30036

[GH] E. Ghys and P. de la Harpe et al., Sur les groupes hyperboliques d'après Mikhael Gromov, Progress in Mathematics, vol. 83, Birkhäuser, Boston, 1990. CMP 91:06

[Gro] M. Gromov, Hyperbolic groups, Essays in group theory, Math. Sci. Res. Inst. Publ., 8, Springer-Verlag, New York and Berlin, 1987, pp. 75-263. MR 89e:20070

[Has] M. Hasumi, Hardy classes on infinitely connected Riemann surfaces, Lecture Notes in Mathematics, vol. 1027, Springer-Verlag, New York and Berlin, 1983. MR 85k:30066

[Hei] M. Heins, A theorem of Wolff-Denjoy type, Complex Analysis, Birkhäuser, Basel, 1988, pp. 81-86. MR 90d:30077

[Hue] H. Hueber, On boundary Harnack principles and poles of extremal harmonic functions, J. Reine Angew. Math. 311/312 (1979), 384-388. MR 81d:31016

[Lár] F. Lárusson, The Martin boundary action of Gromov hyperbolic covering groups and applications to Hardy classes, International J. Math. 6 (1995), no. 4, 601-624. CMP $95: 15$

Department of Mathematics, Purdue University, West Lafayette, Indiana 47907

Current address: Department of Mathematics, University of Western Ontario, London, Ontario, Canada N6A 5B7

E-mail address: Larusson@uwo.ca 